\title{
WHITE MATTER MULTI-RESOLUTION SEGMENTATION USING FUZZY SET THEORY
}

\author{
${ }^{*}$ A. Delmonte ${ }^{1,2},{ }^{*}$ C. Mercier ${ }^{1,3}$, J. Pallud ${ }^{4}$, I. Bloch ${ }^{1,2}$, P. Gori ${ }^{1}$ \\ ${ }^{1}$ LTCI, Télécom ParisTech, University Paris-Saclay, Paris, France \\ ${ }^{2}$ IMAG2 Laboratory, Imagine Institute, Paris, France \\ ${ }^{3}$ LIX, École Polytechnique, Palaiseau, France \\ ${ }^{4}$ Neurosurgery Department, Sainte-Anne Hospital, Paris, France
}

\begin{abstract}
The neural architecture of the white matter of the brain, obtained using tractography algorithms, can be divided into different tracts. Their function is, in many cases, still an object of study and might be affected in some syndromes or conditions. Obtaining a reproducible and correct segmentation is therefore crucial both in clinics and in research. However, it is difficult to obtain due to the huge number of fibers and high inter-subject variability. In this paper, we propose to segment and recognize tracts by directly modeling their anatomical definitions, which are usually based on relationships between structures. Since these definitions are mainly qualitative, we propose to model their intrinsic vagueness using fuzzy spatial relations and combine them into a single quantitative score mapped to each fiber. To cope with the high redundancy of tractograms and ease interpretation, we also take advantage of a simplification scheme based on a multi-resolution representation. This allows for an interactive and real-time navigation through different levels of detail. We illustrate our method using the Human Connectome Project dataset and compare it to other well-known white matter segmentation techniques.
\end{abstract}

Index Terms - Brain, White matter, Tractography, Segmentation, IFOF, UF, Spatial Fuzzy Sets, Multi-resolution

\section{INTRODUCTION}

It is of interest for clinicians and researchers to isolate precise white matter tracts of the brain for surgical planning or to test hypotheses related to a certain condition. These tracts can be reconstructed as bundles of 3D polylines, called tractograms, using tractography algorithms from diffusion MRI scans. Each polyline, called fiber, estimates the trajectory of a large group of axons. Segmenting white matter tractograms into anatomically relevant and reproducible tracts is a difficult task due to the huge number of fibers - up to millions the presence of spurious fibers and the high inter-subject variability. Furthermore, the anatomical definition of a tract is usually qualitative and vague since it is based on spatial relationships such as "anterior to" or "close to" with respect to other anatomical structures.

The most common technique for identifying a tract is to manually delineate Regions of Interest (ROIs) and select (or exclude) the fibers that pass through them [1]. This method is tedious, timeconsuming and not easily reproducible, especially for tracts with convoluted trajectories [2]. Another technique is based on the transfer of manually segmented ROIs from one (or multiple) training images to test subjects via non-linear deformations [2]. The resulting segmentation highly depends on the quality of the registration which

${ }^{*}$ A. Delmonte and C. Mercier contributed equally to this work might not be accurate when training and test images do not share the same anatomical topology (e.g. due to a tumor or illness). A third class of methods is based on clustering algorithms where clusters are usually classified into anatomical tracts using a labelled atlas or training subjects [3, 4, 5, 6]. These methods are usually computationally demanding, depend on user-tuned parameters (e.g. size or number of clusters) and the atlases are usually estimated from a small population of healthy subjects. With a different perspective, in [7] authors proposed a query language (WMQL) to interactively define and segment anatomical tracts employing simple spatial relationships and logical operations. This method is fast and easy to use but the shape of the resulting tracts can vary among subjects since WMQL is based on binary relations and bounding boxes.

Here, we also propose to directly model qualitative anatomical definitions and use them into a segmentation algorithm, but within a richer framework, by representing the inherent imprecision of the anatomical definitions using the theory of fuzzy sets [8]. All qualitative relationships are eventually summarized into a single quantitative "anatomical coherence score" (ACS) which is assigned to every fiber of the tractogram. We also take advantage of a recently proposed parsimonious and multi-resolution representation for white matter tractograms [9]. The user can navigate through different levels of resolution in real-time. This eases the interpretation, especially when using large tractograms composed of millions of fibers.

\section{METHODS}

Modeling spatial relations Qualitative anatomical definitions of white matter tracts are mostly based on spatial relations with respect to anatomical structures (e.g. "anterior to the hippocampus"). Translating these definitions into operational algorithms calls for mathematical models representing the intrinsic vagueness of the definitions. To this end, we rely on fuzzy set theory and on the spatial relation models proposed in [8]. The general idea is to define for each point in the space the degree to which it satisfies a given relation with respect to a reference object (i.e. an anatomical structure). First, we explain how we model directional relations, such as "in direction $\alpha$ with respect to a reference structure $R$ ". Let $\overrightarrow{u_{\alpha}}$ be a unit vector pointing in direction $\alpha$. We define a fuzzy spatial region as a membership function $\mu_{\alpha}^{R}(P)$ which assigns to every point $P$ in the space the degree of being in direction $\alpha$ with respect to $R$. In 2D, for any point $P$, let $\beta\left(\overrightarrow{u_{\alpha}}, \overrightarrow{Q P}\right)$ be the angle between $\overrightarrow{u_{\alpha}}$ and $\overrightarrow{Q P}$, where $Q$ is a point in $R$, and $\beta_{\text {min }}$ is the minimum angle among all points $Q$. We define:

$$
\mu_{\alpha}^{R}(P)=\max \left(0, g\left(\beta_{\text {min }}\right)\right), \quad g\left(\beta_{\text {min }}\right)=1-\frac{2 \beta_{\text {min }}}{\pi}
$$


This means that $\mu_{\alpha}^{R}(P)$ continuously decreases from 1 to 0 , being equal to 1 when $P$ is exactly in direction $\alpha$ with respect to $R$ and 0 when it is in the opposite direction. Note that other decreasing functions $g$ could be used. In 3D, the direction is represented by two angles and computing $\mu_{\alpha}^{R}$ from Eq. 1 might be expensive. To address this issue, in [10] a more efficient approach was proposed by expressing $\mu_{\alpha}^{R}$ as a fuzzy dilation. One can show that:

$$
\mu_{\alpha}^{R}(P)=D_{\nu}(R)(P)=\max _{Q \in R} \nu(P-Q)
$$

where $D$ is a fuzzy dilation, expressed here for a binary segmentation $R$, by the fuzzy structuring element $\nu(P)=\max (0$, $\left.g\left(\beta\left(\overrightarrow{u_{\alpha}}, \overrightarrow{O P}\right)\right)\right)$ where $O$ is the origin of the space. Using this approach, we model the directions anterior, posterior, superior, inferior, right and left as well as the relations "lateral" and "medial", which are commonly used in the neuro-anatomical literature. For these two last relations, we use as reference the mid-sagittal plane which is automatically detected using the method described in [11].

In the literature, a white matter tract is usually described as a logic combination of several relations, using operators such as $A N D$ and $O R$. The proposed fuzzy models of spatial relations are combined using fuzzy $A N D$ (using t-norms) and fuzzy $O R$ (using tconorms). Here, we use the minimum for $A N D$ and the maximum for $O R$, computed voxel-wise. The complete list of definitions is represented as an abstract syntax tree, that is collapsed in a hierarchical order to eventually compute the final fuzzy region satisfying the combination of the relations. A membership value $\mu^{*}$ describing the degree of satisfaction of the combined relations is computed for every point $P$ in the space (i.e. every voxel). A fuzzy score FS is then assigned to every fiber of a tractogram, which is computed as the weighted average of the membership values $\mu^{*}$ of the voxels the fiber passes through. Weights are computed as the proportion of the length of the fiber within each voxel.

In addition to the relative directions, we also model another common anatomical definition about the location of the tract terminations (e.g. "fibers terminate in temporal lobe"). Let $f$ be one of the endpoints of a fiber and $M$ the region of the ending area, we define the degree of rightness as: $\mathrm{EP}=\max _{m \in M} \exp -\frac{\|f-m\|_{2}^{2}}{\lambda^{2}}$, where $\lambda$ is a fixed parameter. When the definition involves only one region, $f$ is the endpoint closer to $M$. Otherwise, when using two ending areas, the fiber orientation is the one minimizing the sum of the distances between the ending points and the regions (each extremity being linked to a different region). Note that, in this work, fibers have been cut at the boundary between white and gray matter. Eventually, all relations are combined together in a conjunctive way: $\mathrm{ACS}=\mathrm{FS} \times \mathrm{EP}$.

Multi-resolution representation Interpreting or choosing a threshold value for ACS might be quite hard when working with tractograms composed of millions of fibers. This is particularly the case for applications demanding a high accuracy like surgical planning. To this end, exploiting our recently presented fiber simplification method [9], we progressively group the most similar streamlines into generalized cylinders with elliptical basis, producing a nested hierarchy of resolution levels, where every level of detail corresponds to the fusion of two fibers (or cylinders). Here, we propose to compare fibers using an extension of the computational model of Weighted Currents $\left(W C_{\text {ext }}\right)$ [12]. Let $X=\left\{x_{i}, \alpha_{i}\right\}$ and $Y=\left\{y_{j}, \beta_{j}\right\}$ be two fibers of $N$ and $M$ segments respectively, where $x_{i}$ and $\alpha_{i}$ (respectively $y_{j}$ and $\beta_{j}$ ) are the centers and tangent vectors of $X$ (respectively $Y$ ), the proposed similarity measure is defined as:

$$
\begin{aligned}
W C_{\text {ext }}= & K_{c}\left(\left|\mathrm{ACS}_{X}-\operatorname{ACS}_{Y}\right|\right) K_{a}\left(\left\|f_{a}-t_{a}\right\|_{2}\right) \\
& K_{b}\left(\left\|f_{b}-t_{b}\right\|_{2}\right)\left|\sum_{i=1}^{N-1} \sum_{j=1}^{M-1} \alpha_{i}^{T} K_{g}\left(\left\|x_{i}-y_{j}\right\|_{2}\right) \beta_{j}\right|
\end{aligned}
$$

where $\mathrm{ACS}_{X}$ and $\mathrm{ACS}_{Y}$ are the ACS values of the two fibers; $f_{a}, f_{b}$ and $t_{a}, t_{b}$ are the corresponding endpoints of $X$ and $Y$ respectively; $K_{a}, K_{b}$ and $K_{g}$ are three Gaussian kernels parametrized by $\sigma_{a}, \sigma_{b}$ and $\sigma_{g}$ respectively and $K_{c}(|A-B|)=1-|A-B|$ is the new kernel proposed here. Two fibers are considered similar, and thus merged together, only if their trajectories are alike, their endpoints are close to each other and their ACS values are similar. We also propose an automatic stopping criterion for the multi-resolution to prevent oversimplification (e.g. a single cylinder). We use the inner product of $\mathrm{Eq} 3$ to compute angles between fibers and cylinder center-lines. Two cylinders/fibers are not merged together if they are almost orthogonal $\left(\right.$ angle $\left.>89^{\circ}\right)$. Fibers that were never merged in the whole process are then considered outliers and discarded. The proposed technique simplifies the geometric representation, preserving at the same time the overall structure of the original tractogram (i.e. shape, connectivity and ACS). Instead of comparing all fibers to each other, which would lead to intractable computations, we rely on a Delaunay tetrahedralization of their extremities. This provides a geometrically well distributed set of adjacency links. The similarity measure in $\mathrm{Eq} 3$ is only computed between adjacent (linked) fibers [9]. Fuzzy scores for cylinders are computed as the weighted average of the membership values $\mu^{*}$ of the voxels the cylinders cross. As for fibers, weights are computed as the proportion of the length of the cylinder inside the voxels. The distance between cylinder terminations and ending regions $M$ is computed as the average distance between the ending surface of the cylinder and the region $M$. We also provide a GUI where the user can navigate in real-time through different levels of detail and at the same time select only the fibers/cylinders with an ACS value above a user-defined threshold.

\section{RESULTS AND DISCUSSION}

Dataset and Numerical aspects Experiments are based on 10 uncinate fasciculus (UF) and 10 inferior fronto-occipital fasciculus (IFOF) segmented from 5 randomly selected healthy subjects of the $H C F^{1}$ dataset. Whole-brain tractograms of one million fibers are estimated with MRTrix $3^{2}$ using a probabilistic algorithm (iFOD2) and the Constrained Spherical Deconvolution model. We separately analyze the two brain hemispheres removing fibers measuring less than $4 \mathrm{~cm}$. In Eq3. we used the value of $6 \mathrm{~mm}$ for $\sigma_{a}, \sigma_{b}$ and $\sigma_{g}$. Gray matter structures are extracted using FreeSurfe ${ }^{3}$ Fuzzy definitions are implemented in Python and the computational time is about $10-15$ s per definition. Multi-resolution is instead implemented in C++, Qt and OpenGL. The computational time depends on the number of fibers $n$ with a complexity of $O(n \log (n))$ [9]. The source code will be publicly available at https://github.com/CorentinMercier/FBTS

Tract modeling Based on both imaging and dissection studies [1, 13 14, 15] and with the help of an experienced neurosurgeon, we define UF and IFOF considering the labels available in FreeSurfer. The

\footnotetext{
${ }^{1}$ https://db.humanconnectome.org

${ }^{2}$ http://www.mrtrix.org

${ }^{3}$ https://surfer.nmr.mgh.harvard.edu

${ }^{4}$ Please note that any gray matter segmentation pipeline could be used
} 


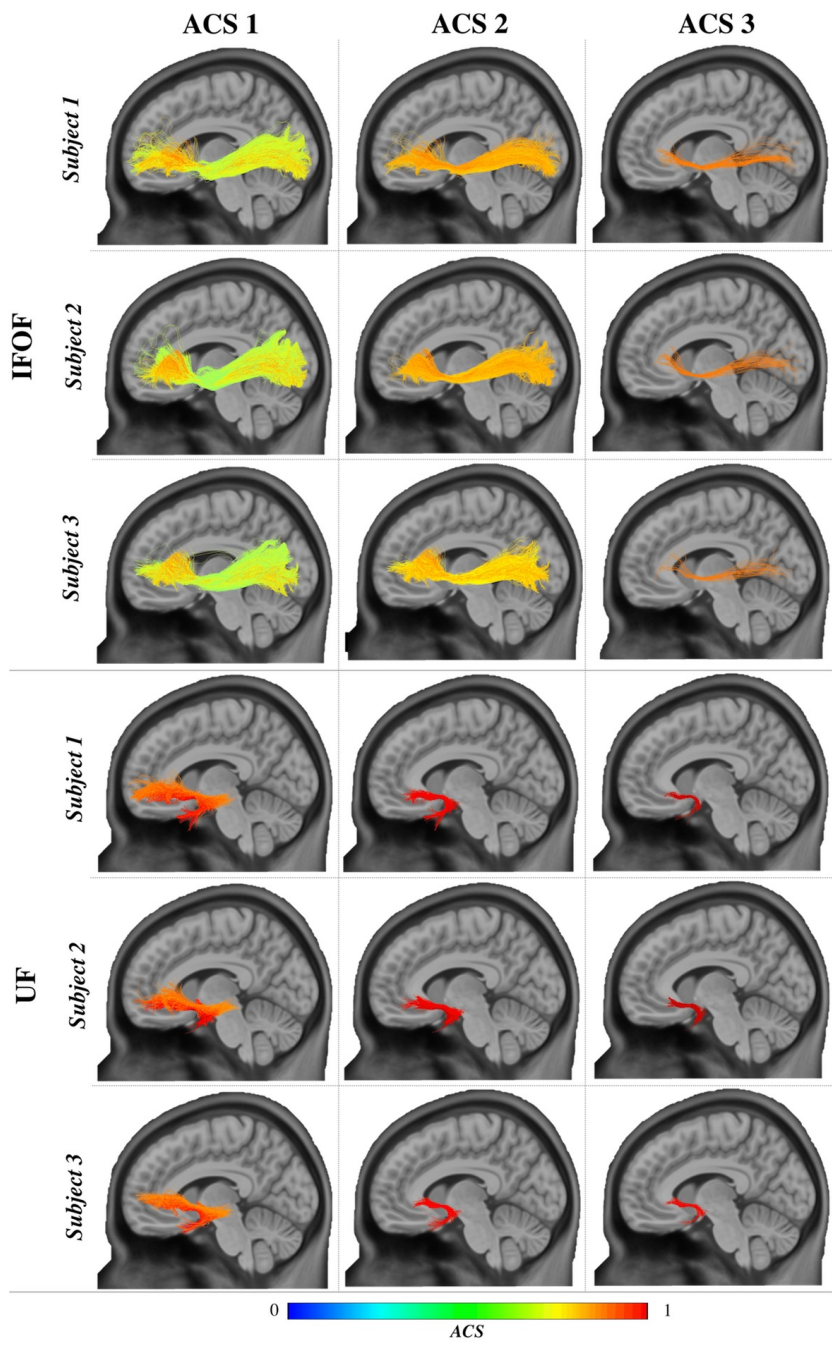

Fig. 1. Segmentation of IFOF and UF bundles of three subjects using three different thresholds for the ACS $(0.5,0.65,0.7$ for the IFOF and $0.7,0.85,0.9$ for the UF respectively). Results are shown on the MNI152 T1w image.

employed definition for the UF is: inferior of putamen AND inferior of claustrum AND anterior of amygdala. Moreover, one endpoint of the fibers should be in the anterior temporal lobe. For the IFOF, we combine together the frontal, occipital and stem definitions as: (internal AND lateral of external capsule AND anterior of hippocampus) OR (anterior AND superior AND lateral of putamen) OR (posterior of putamen AND posterior AND superior of hippocampus). Furthermore, fibers should have one endpoint in the occipital lobe and the other one in the orbital and inferior frontal gyrus.

We also propose to model the occipital lobe using the following rule: the cuneus, lateral-occipital section and pericalcarine regions are all considered part of the occipital lobe. Then, we find a plane $P$ perpendicular to the principal direction of the hippocampus and we move it along the posterior direction until it reaches one of the three previous regions. All voxels composing the fusiform or lingual label and lying posterior to the plane $P$ define the complementary part of the occipital lobe.

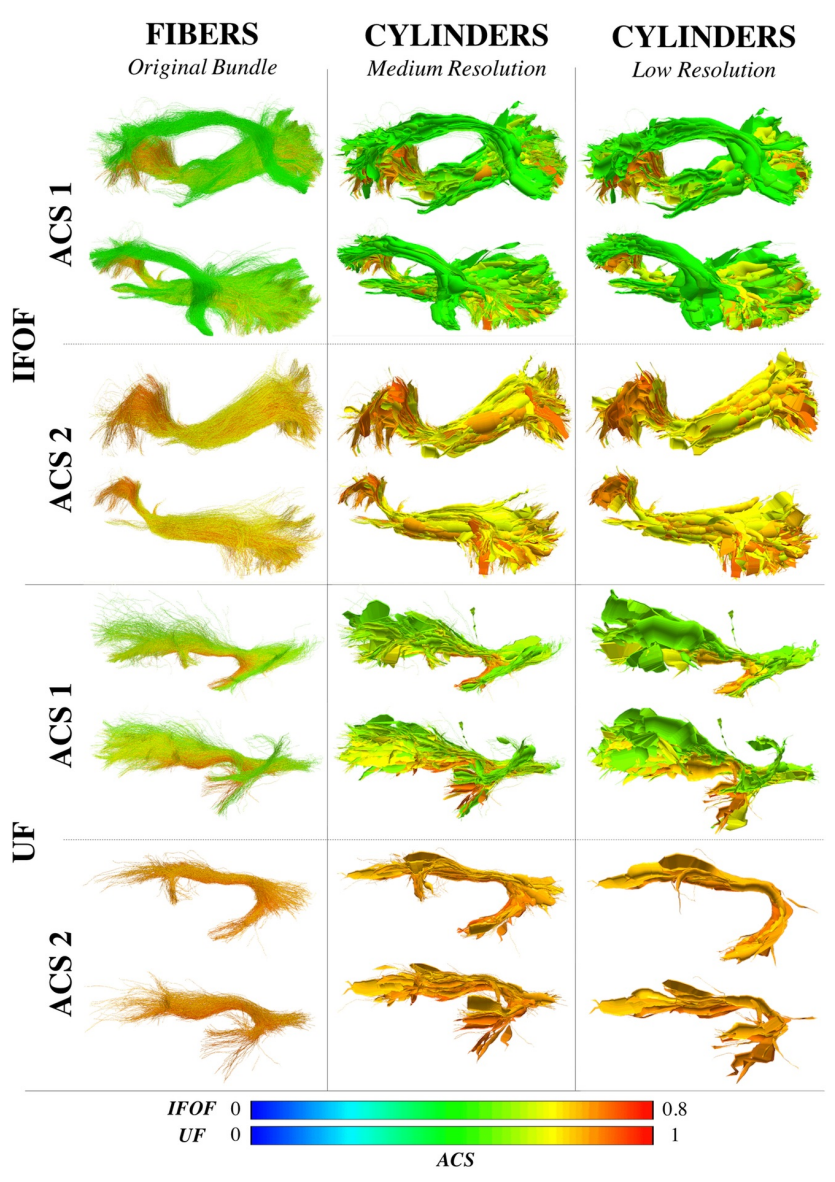

Fig. 2. IFOF and UF bundles visualized at three different resolution levels and segmented with two different ACS thresholds $(0.4,0.57$ for the IFOF and 0.6, 0.81 for the UF respectively).

Experiments In Fig.11, we show the segmentation of the IFOF and UF for three subjects using three different ACS thresholds. It can be noticed that results are very similar among subjects even when varying the thresholds. Reproducibility has also been observed at other thresholds.

In Fig. 2 different levels of resolution are shown for both IFOF and UF using two thresholds for the ACS. The proposed parsimonious model based on generalized cylinders eases the interpretation of a tract by giving a summarized representation of the original fibers. The structure of the original bundle is more visible and recognizable at a lower resolution (2nd and 3rd columns). Furthermore, the proposed GUI allows the user to navigate in real-time through different levels of resolution and display fibers/cylinders with ACS higher than a selected threshold. Using it, our clinical colleagues were able to analyze the structures of the tracts at different resolutions and thresholds and choose an optimal one (ACS2 in Fig. 1] and Fig. 2.

In Fig. 3 we compare the results of the proposed method with the ones obtained using a manual segmentation, delineated following [1, 15], and the default WMQL queries [7]. Although often considered the most reliable approach, the manual segmentation cannot be assumed as a ground truth (only a post-mortem study would be) [1] 16]. We thus decided to quantitatively compare the three meth- 


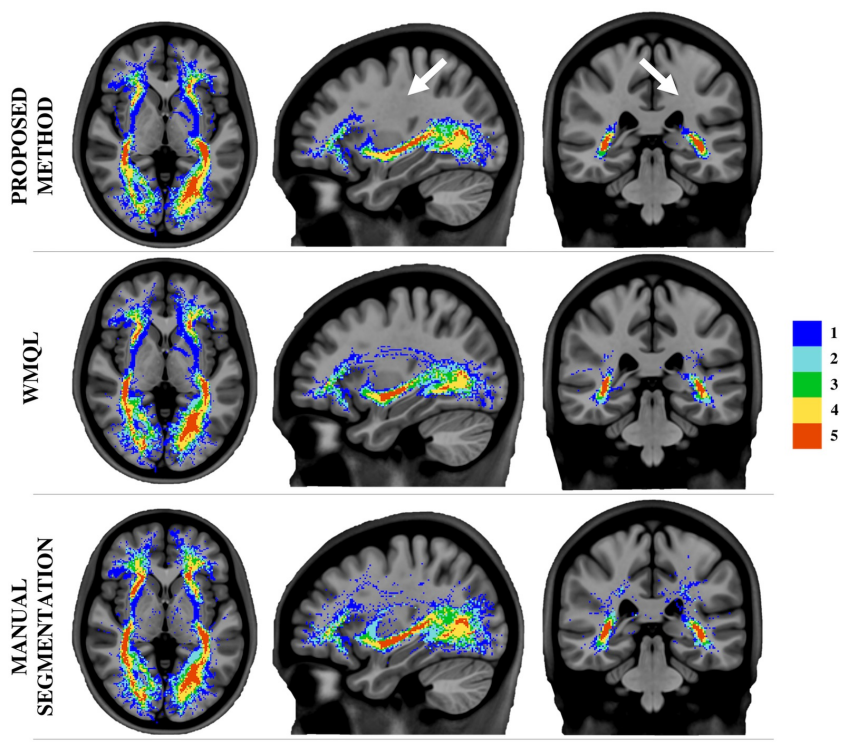

Fig. 3. Reproducibility analysis carried on five subjects from the $H C P$ dataset. A colormap encodes, for each voxel of the image, the number of subjects that have at least one of the segmented IFOF fibers passing through it. The areas showing a smaller fiber dispersion with our method are highlighted using a white arrow.

ods by looking at their reproducibility. We make the hypothesis that spurious fibers, namely fibers with atypical trajectories with respect to the fibers of all analyzed subjects, should be considered as outliers. Both WMQL and manual based segmentations show a greater dispersion in the pathways of the fibers, and thus less reproducible results, compared to the proposed method. The percentage of whitematter voxels containing the IFOF fibers of at least one subject decreases from the $15 \%$ of the manual segmentation, to $9 \%$ in WMQL, to the achieved $6 \%$. This is mainly due to the fact that our approach produces more compact and consistent segmentations where there are fewer voxels with fibers belonging to only one subject.

Note that, for both hemispheres, the computational time for the IFOF (resp. UF) was $\sim 100$ s (resp. $\sim 25$ s) for the proposed method, $\sim 300$ s (resp. $\sim 240$ s) for WMQL and $\sim 900$ s (resp. $\sim 900$ s) for the manual segmentation, respectively.

\section{CONCLUSIONS}

We proposed a segmentation algorithm for white matter that produces anatomically-relevant and reproducible tracts. Differently from most segmentation algorithms, we directly model the qualitative anatomical definitions of the tracts using the fuzzy set theory. This makes it possible to cope with the inherent imprecision of the anatomical definitions and to combine them into a single quantitative value (ACS) which summarizes the degree of satisfaction of all relations. To ease interpretation when working with millions of fibers, we also proposed to adapt a recent simplification algorithm [9] to our segmentation problem. It produces a multi-resolution representation and provides a GUI where the user can navigate in real-time through different levels of resolution. This helped our clinical colleagues to better understand the structure of the bundles and find an optimal ACS threshold for the segmentation of IFOF and UF. Our preliminary results showed that the proposed method is more reproducible and consistent than using a manual segmentation or WMQL [7]. We plan to extend the proposed technique to other tracts and test it on more subjects.

Acknowledgements This research was supported by Labex DigiCosme (project ANR11LABEX0045DIGICOSME) as part of the program Investissement d'Avenir Idex ParisSaclay (ANR11IDEX000302).

\section{REFERENCES}

[1] S. Wakana et al., "Reproducibility of quantitative tractography methods applied to cerebral white matter," NeuroImage, vol. 36, pp. 630-644, 2007.

[2] Y. Zhang et al., "Atlas-guided tract reconstruction for automated and comprehensive examination of the white matter anatomy," NeuroImage, vol. 52, no. 4, pp. 1289-1301, 2010.

[3] L. O'Donnell et al., "Automatic Tractography Segmentation Using a High-Dimensional White Matter Atlas," IEEE TMI, vol. 26, no. 11, pp. 1562-1575, 2007.

[4] P. Guevara et al., "Automatic fiber bundle segmentation in massive tractography datasets using a multi-subject bundle atlas," NeuroImage, vol. 61, no. 4, pp. 1083-1099, 2012.

[5] G. Prasad et al., "Automatic clustering and population analysis of white matter tracts using maximum density paths," NeuroImage, vol. 97, pp. $284-295,2014$.

[6] E. Garyfallidis et al., "Recognition of white matter bundles using local and global streamline-based registration and clustering," NeuroImage, vol. 170, pp. 283 - 295, 2018.

[7] D. Wassermann et al., "The white matter query language: a novel approach for describing human white matter anatomy," Brain Struct. Func., vol. 221, no. 9, pp. 4705-4721, 2016.

[8] I. Bloch, "Fuzzy spatial relationships for image processing and interpretation: a review," Image and Vision Computing, vol. 23, no. 2, pp. 89-110, 2005.

[9] C. Mercier et al., "Progressive and Efficient Multi-Resolution Representations for Brain Tractograms," in EG VCBM, 2018.

[10] I. Bloch, "Fuzzy Relative Position between Objects in Image Processing: a Morphological Approach," IEEE Transactions on Pattern Analysis and Machine Intelligence, vol. 21, no. 7, pp. 657-664, 1999.

[11] A. Tuzikov et al., "Evaluation of the Symmetry Plane in 3D MR Brain Images," Pattern Recognition Letters, vol. 24, no. 14, pp. 2219-2233, 2003.

[12] P. Gori et al., "Parsimonious Approximation of Streamline Trajectories in White Matter Fiber Bundles," IEEE TMI, vol. 35, no. 12, pp. 2609-2619, 2016.

[13] S. Sarubbo et al., "Frontal terminations for the inferior frontooccipital fascicle: anatomical dissection, dti study and functional considerations on a multi-component bundle," Brain Structure and Function, vol. 218, no. 1, pp. 21-37, 2013.

[14] U. Ebeling et al., "Topography of the uncinate fascicle and adjacent temporal fiber tracts," Acta Neurochirurgica, vol. 115, no. 3, pp. 143-148, 1992.

[15] M. Catani et al., "A diffusion tensor imaging tractography atlas for virtual in vivo dissections," Cortex, vol. 44, no. 8, pp. 11051132, 2008.

[16] V. J. Sydnor et al., "A comparison of three fiber tract delineation methods and their impact on white matter analysis," NeuroImage, vol. 178, pp. 318 - 331, 2018. 